Nijs, J., Meeuw, M., Heins, M., Knoop, H., Moorkens, G., Bleijenberg, G. Kinesiophobia, catastrophizing and anticipated symptoms before stair climbing in chronic fatigue syndrome: an experimental study. Disability and Rehabilitation: 2012, 34(15), 1299-1305

\begin{tabular}{|l|l|}
$\begin{array}{l}\text { Postprint } \\
\text { Version }\end{array}$ & 1.0 \\
\hline Journal website & http://informahealthcare.com/doi/abs/10.3109/09638288.2011.641661 \\
\hline Pubmed link & $\underline{\text { http://www.ncbi.nlm.nih.gov/pubmed/22324510 }}$ \\
\hline DOI & $10.3109 / 09638288.2011 .641661$ \\
\hline
\end{tabular}

This is a NIVEL certified Post Print, more info at http://www.nivel.eu

\title{
Kinesiophobia, catastrophizing and anticipated symptoms before stair climbing in chronic fatigue syndrome: an experimental study
}

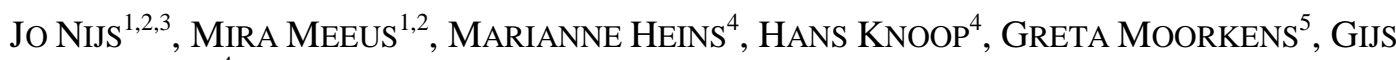 \\ BLEIJENBERG $^{4}$ \\ ${ }^{1}$ Department of Human Physiology, Faculty of Physical Education \& Physiotherapy, Vrije \\ Universiteit Brussel, Brussels, Belgium \\ ${ }^{2}$ Division of Musculoskeletal Physiotherapy, Department of Health Care Sciences, Artesis \\ University College Antwerp, Antwerp, Belgium \\ ${ }^{3}$ Department of Physical Medicine and Physiotherapy, University Hospital Brussels, \\ Brussels, Belgium \\ ${ }^{4}$ Expert Centre for Chronic Fatigue, Radboud University Nijmegen Medical Centre, \\ Nijmegen The Netherlands \\ ${ }^{5}$ Reference Centre for Chronic Fatigue Syndrome, Department of Internal Medicine, \\ University Hospital Antwerp, Antwerp, Belgium
}

Purpose: Kinesiophobia and catastrophizing are frequent among people with chronic fatigue syndrome (CFS). This study was aimed at examining (1) whether kinesiophobia, anticipated symptoms and fatigue catastrophizing are related to stair climbing performance in people with CFS; and (2) whether kinesiophobia and fatigue catastrophizing are related to daily physical activity in CFS. Method: Patients with CFS filled in a set of questionnaires, performed a physical demanding task (two floors stair of climbing and descending) with pretest and post-test heart rate monitoring and immediate post-stair climbing symptom assessment. Real-time activity monitoring was used between the baseline and second assessment day (7 days later). Results: Kinesiophobia and fatigue catastrophizing were strongly related ( $\rho=0.62$ and 0.67 , respectively) to poorer stair climbing performance (i.e. more time required to complete the threatening activity). Kinesiophobia and fatigue catastrophizing were unrelated to the amount of physical activity on the first day following stair climbing or during the seven subsequent days. Conclusion: These findings underscore the importance of kinesiophobia and fatigue catastrophizing for performing physical demanding tasks in everyday life of people with CFS, but refute a cardinal role for kinesiophobia and fatigue catastrophizing in determining daily physical activity level in these patients. 
Nijs, J., Meeuw, M., Heins, M., Knoop, H., Moorkens, G., Bleijenberg, G. Kinesiophobia, catastrophizing and anticipated symptoms before stair climbing in chronic fatigue syndrome: an experimental study. Disability and Rehabilitation: 2012, 34(15), 1299-1305

\section{Implications for Rehabilitation}

People with chronic fatigue syndrome (CFS) can easily perform stair climbing, a daily physical activity perceived by themselves as threatening, without triggering symptom flares. This is important as it can be used clinically to convince people with CFS of undertaking such a threatening task during treatment programs such as graded activity or graded exposure.

The finding that kinesiophobia and fatigue catastrophizing are strongly related to stair climbing performance in CFS underscores the importance of restructuring the beliefs of these patients about the relationship between activity and symptoms. This should be a key component of the early stages of rehabilitation for people with CFS. In rehabilitation practice, diminishing kinesiophobia and fatigue catastrophizing in patients with CFS appears only relevant for targeting physical activities that are perceived as threatening (e.g. stair climbing), and not for increasing work-related or social (physical) activities.

Clinicians can use the Tampa Scale Kinesiophobia version CFS for assessing the fear of patients with CFS to exacerbate their symptoms (including pain, fatigue and brain fog) due to physical activities.

Clinicians can use the Tampa Scale fatigue for assessing the fear of patients with CFS to exacerbate their fatigue level due to physical activities.

\section{INTRODUCTION}

In people with chronic fatigue syndrome (CFS), too vigorous exercise [1-3] or a sudden increase in activity [4] frequently triggers an increase in symptoms. This severe exacerbation of symptoms following exercise, as seen in CFS patients, is one of the core features of the illness [5,6]. Thus, it seems natural for people with CFS to develop fear of performing physical demanding tasks (i.e. exercises or activities that trigger a symptom increase), and consequently avoidance behaviour towards physical activity. Kinesiophobia, a specific kind of fear-avoidance behaviour, is defined as "an excessive, irrational and debilitating fear of physical movement and activity resulting from a feeling of vulnerability to painful injury or re-injury" [7].

Kinesiophobia has been reported to be a common feature of people with CFS [8-10], and some evidence supportive of its clinical importance to CFS patients has been provided $[8,10]$. Others interpreted the lack of a difference in physical activity in anticipation of an exercise test, both on the day of and the day before the test, as a lack of "exercise phobia" in CFS patients [11]. Thus, further research of the exact role of kinesiophobia in relation to physical activity in people with CFS is warranted. Anticipated symptoms before physical activity (i.e. the subject's predicted symptoms during a future physical activity) might influence a patient's physical performance, daily physical activity level, as well as beliefs [3]. Patients with high degrees of anticipated symptom increases before a particular physical demanding task may avoid the task or perform it less vigorously. As a consequence, patients might even become less physically active. There is some evidence suggesting that fatigue severity is related to actual physical activity level and high fatigue expectations are related to low physical activity levels in people with CFS [12]. This suggests that people with CFS try to prevent symptoms by avoiding physical activity. This view is 
Nijs, J., Meeuw, M., Heins, M., Knoop, H., Moorkens, G., Bleijenberg, G. Kinesiophobia, catastrophizing and anticipated symptoms before stair climbing in chronic fatigue syndrome: an experimental study. Disability and Rehabilitation: 2012, 34(15), 1299-1305

further supported by the observation that high levels of fear of movement are related to low daily physical activity and high disability levels [10].

Unfortunately, previous studies examined fear of movement in CFS patients using exercise stress tests [8-11], which is unlikely to have high ecological validity. Therefore, the present study was undertaken to examine the role of kinesiophobia in relation to a physically demanding task for people with CFS, and one which with everyone is familiar (i.e. stair climbing). People with CFS should be able to accurately anticipate symptom changes following this task. It is hypothesized that low kinesiophobia levels and low symptom expectancies correspond to better performance during stair climbing (e.g. higher speed) in people with CFS. Besides kinesiophobia and symptom expectancies, other factors may affect stair climbing performance in people with CFS. Catastrophizing, defined as a lack of confidence and a tendency to expect negative outcomes, is currently viewed as a multidimensional construct comprising elements of rumination, magnification and helplessness [13]. Petrie et al. [14] found that among self-diagnosed CFS patients, catastrophizers reported greater levels of disability. We previously reported that pain catastrophizing (i.e. helplessness and rumination rather than magnification) predicts bodily pain in female CFS patients, even after controlling for depression [15]. The same study revealed that physiological exercise performance in female CFS patients is related to both pain catastrophizing and pain severity but not to depression [15]. However, limited data addressing fatigue catastrophizing in patients with CFS are currently available. Hence, here we hypothesize that fatigue catastrophizing accounts in part for stair climbing performance and even daily physical activity level in people with CFS.

In summary, the study aims at examining (1) whether stair climbing performance (i.e. speed) is associated with symptom expectancies, kinesiophobia and fatigue catastrophizing in people with CFS and (2) whether kinesiophobia and fatigue catastrophizing are related to daily physical activity in CFS. This report entails the Belgian part of an international multi-centre study to examine the role of kinesiophobia and catastrophizing in people with CFS. We previously reported that performance during physical activity is directly influenced by symptom expectancies and focusing on symptoms in Dutch people with CFS [16]. Here, we replicate our previous findings and extend them with a 7-day follow-up of symptom severity and real-time physical activity monitoring following stair climbing.

\section{MATERIALS AND METHODS}

\section{Study design}

On the first day of the study, participants provided written informed consent and rated their expected fatigue and pain levels following 10 tasks (a 10-minute walk on a flat surface, 15-minute ironing, a 2-minute run, 30-minute reading, 2 floors of stair climbing and descending, washing dishes, preparing a meal, driving a car for 30 minutes, 30-minute computer work and 15-minute gardening) on visual analogue scales. Next, they completed the Medical Outcomes Short Form 36 Health Status Survey (SF-36), Checklist Individual Strength (CIS), CFS Symptom List, shortened version of the Tampa scale for fear of fatigue (TS-fatigue), Tampa scale kinesiophobia version CFS (TSK-CFS), Jacobson-Fatigue Catastrophizing Scale (JFCS) and rated two visual analogue scales (for the assessment of the severity of two 
Nijs, J., Meeuw, M., Heins, M., Knoop, H., Moorkens, G., Bleijenberg, G. Kinesiophobia, catastrophizing and anticipated symptoms before stair climbing in chronic fatigue syndrome: an experimental study. Disability and Rehabilitation: 2012, 34(15), 1299-1305

main symptoms: fatigue and pain). The use of two different measures for assessing kinesiophobia in people with CFS (the TS-fatigue and TSK-CFS) was chosen for comparison of the validity of both measures. Afterward, participants performed the physical demanding task (two floors stair climbing and descending) with pre-test and post-test heart rate monitoring and immediate post-stair climbing symptom assessment (visual analogue scales for fatigue and pain) (Figure 1). Real-time activity monitoring (accelerometry) was used between the baseline and second assessment day (7 days later). For monitoring symptom severity changes following the stair climbing experiment, participants filled in the CFS Symptom List exactly 24 hours post-stair climbing. On day 8 of the study, participants completed the CFS Symptom List, TS-fatigue and TSK-CFS.

\section{[FIGURE 1]}

\section{Participants}

Women with CFS $(\mathrm{n}=21)$ within the age range of $24-60$ years (mean age $=44.7 \pm 10.5$; median illness duration 84 months) were recruited from the Reference Centre for Chronic Fatigue Syndrome of the University Hospital of Antwerp, Belgium. The descriptive statistics of the study participants are displayed in Table I. All participants fulfilled the Centre for Disease Control and Prevention criteria for CFS [35]. An extensive medical evaluation prior to study participation was part of the diagnostic procedure. In light of the physical demanding task (stair climbing), all participants having heart or knee disorders were excluded. Our primary outcome was the association between kinesiophobia (i.e. the TSK-CFS total score) and performance during stair climbing. To obtain a correlation coefficient of at least 0.60 and a power of $0.90(1-\beta)$, a study sample of at least 20 participants is required [17].

\section{[TABLE 1]}

\section{Questionnaires}

The CFS Symptom List is a self-reported measure for assessing symptom severity in CFS patients. To assess the severity of the 19 symptoms included in the CFS Symptom List, visual analogue scales $(100 \mathrm{~mm})$ are used. Psychometric work supporting the use of the Dutch and French versions of the CFS Symptom List has been published $[18,19]$.

The SF-36 assesses functional status and well-being or quality of life [20]. The SF36 has been documented to have good reliability and validity in a wide variety of patient populations [20-22], and it is the most frequently used measure to assess functional status in CFS research [23].

The CIS aims at assessing fatigue severity, concentration difficulties, motivation problems and the level of self-reported physical activity [24]. Higher scores on the CIS correspond to more severe fatigue, concentration difficulties, problems with motivation and a low level of physical activity. Its psychometric properties are well established [24-26]. 
Nijs, J., Meeuw, M., Heins, M., Knoop, H., Moorkens, G., Bleijenberg, G. Kinesiophobia, catastrophizing and anticipated symptoms before stair climbing in chronic fatigue syndrome: an experimental study. Disability and Rehabilitation: 2012, 34(15), 1299-1305

The TSK-CFS is a modification of the Tampa scale for kinesiophobia, a selfassessment tool for the measurement of pain-related fear of movement [27], in which the word "pain" has been replaced by "my symptoms" [10]. Extensive work supportive of the internal consistency (Cronbach's $\alpha=0.80$ ), congruent validity, convergent validity, content validity and test-retest reliability of the Dutch and French versions of the TSK-CFS in CFS patients has been provided $[10,18]$. Fatigue-related kinesiophobia was measured with the TS-fatigue $[8,16]$. The TSfatigue is a scale based on the Tampa scale kinesiophobia in which the word "pain" has been replaced with the word "fatigue" [8]. The scale has been translated in Dutch [16] and has been used in a large population of CFS patients and patients with other conditions. Based on a varimax rotated factor analysis, we developed a shortened scale (TS-fatigue-S) consisting of eight items (items 1, 3, 6, 7, 10, 11, 14 and 17 of the original TS-fatigue-S) with a total score ranging from 8 to 36. Reliability of the new scale was assessed in 195 patients with a diagnosis of CFS and was sufficient (Cronbach's $\alpha=0.80$ ).

The J-FCS is a 10-item scale measuring fatigue-related catastrophizing [28]. Each item consists of a 5-point Likert scale on which patients rate how often each item is true for them when they experience fatigue $(1=$ "never true" to $5=$ "all of the time true"). A total score is computed by adding the items and dividing them by 10 . In a sample of women with breast cancer, Cronbach's $\alpha$ was 0.85 [28].

\section{Stair climbing}

Participants were instructed to climb two floors of stairs $(4 \times 13$ steps, height/depth $16 \mathrm{~cm} / 28 \mathrm{~cm}$ ) and to descend to their starting position. They were instructed to perform the activity in a comfortable way (i.e. self-paced) but without resting periods within the task (e.g. participants were instructed not to wait on top of the stairs prior to descending). A heart rate monitor (Polar, FS 1, Polar Electra Belgium n.v.) was used throughout the stair climbing.

\section{Real-time physical activity monitoring}

The Actical (Mini Mitter, Bend, OR, USA) accelerometer was used for real-time monitoring of physical activity. The Actical accelerometer has an omnidirectional sensor and is capable of measuring movement in one plane. It is worn at the wrist. The sensor functions via a cantilevered rectangular piezoelectric bimorph plate and seismic mass, and it is capable of detecting movements in the $0.5-3 \mathrm{~Hz}$ range.

Voltage generated by the sensor is amplified and filtered via analogue circuitry. The amplified and filtered voltage is passed into an analogue to a digital converter, and the process is repeated 32 times per second ( $32 \mathrm{~Hz}$ ). The resulting 1-second value is divided by four, and then added to an accumulated activity value (activity counts) for the epoch. The Actical is the smallest accelerometer available $(28 \times 27 \times 10 \mathrm{~mm}, 17$ g) and is water resistant. Accelerometers are the gold standard for measuring physical behaviour during daily activities. The Actical accelerometer has been used in scientific research and has shown to be valid for the real-time assessment of physical behaviour [29]. For the present study, the monitors were initialized to save data in 1-minute intervals (epochs) and the accelerometer was worn by the patients 24 hours per day. The following physical activity variables were processed: the amount of daily physical activity, the amount of physical activity (i.e. activity 
Nijs, J., Meeuw, M., Heins, M., Knoop, H., Moorkens, G., Bleijenberg, G. Kinesiophobia, catastrophizing and anticipated symptoms before stair climbing in chronic fatigue syndrome: an experimental study. Disability and Rehabilitation: 2012, 34(15), 1299-1305

counts) during the peak activity hour for each day (identified as the hour with the highest number of activity counts), peak ratio for each day (counted as the amount of physical activity during the peak activity hour/mean amount of physical activity on that day), mean time spent doing light activity $(<3$ metabolic equivalent of tasks (METs)), and the time spent doing sedentary ( $<1$ MET), moderate (3-6 METs) or vigorous activity (>6 METs).

\section{Statistical analysis}

Data were analysed using SPSS version 12.0 for Windows (SPSS Inc. Headquarters, Chicago, IL, USA). A conservative approach to the statistical analysis (i.e. nonparametric Spearman rank correlation analyses) was chosen. To examine the real threat value of stair climbing to people with CFS, the mean expected symptom increase following stair climbing was compared with the mean expected symptom increase following the remaining tasks using the non-parametric Wilcoxon signed rank test. Likewise, changes in symptom severity following stair climbing were assessed using the non-parametric Wilcoxon signed rank test. The level of significance was set at $\mathrm{p}<0.05$, but when appropriate adjusted to the 0.01 level to account for potential type I errors (e.g. when using multiple correlations).

\section{RESULTS}

\section{Threat control}

The mean expected pain increase following stair climbing was higher than the mean expected pain increase following the 10 remaining tasks (a 10-minute walk on a flat surface, 15-minute ironing, a 2-minute run, 30-minute reading, 2 floors of stair climbing and descending, washing dishes, preparing a meal, driving a car for 30 minutes, 30 -minute computer work and 15 -minute gardening) (49.3 \pm 27.4 vs. $34.5 \pm 19.0 ; \mathrm{p}=0.04)$. There was a trend towards a higher expected fatigue increase following stair climbing when compared to the expected fatigue increase following the remaining tasks $(59.9 \pm 29.0$ vs. $46.2 \pm 18.5 ; \mathrm{p}=0.079)$. Hence, stair climbing seems to be more threatening than the remaining tasks to the participants with CFS studied here.

\section{Does symptom severity change following stair climbing in CFS?}

There was no change in symptom severity from baseline to 24 hours or 7 days poststair climbing $(\mathrm{p}>0.01)$. This accounts for the total score and the 19 individual symptoms included in the CFS Symptom List.

\section{Are symptom expectancies and kinesiophobia related to stair climbing performance in CFS?}

Table II displays the descriptive statistics of the kinesiophobia and stair climbing data. Symptom expectancies were unrelated to kinesiophobia, fatigue catastrophizing or stair climbing performance (Table III). Likewise, the differences between anticipated and experienced fatigue/pain increasing following stair climbing were unrelated to kinesiophobia, fatigue catastrophizing or stair climbing performance. However, kinesiophobia (i.e. both the TSK-CFS and TS-fatigue total scores) and 
Nijs, J., Meeuw, M., Heins, M., Knoop, H., Moorkens, G., Bleijenberg, G. Kinesiophobia, catastrophizing and anticipated symptoms before stair climbing in chronic fatigue syndrome: an experimental study. Disability and Rehabilitation: 2012, 34(15), 1299-1305

fatigue catastrophizing were strongly related $(\rho=0.6)$ to stair climbing performance (i.e. the time required to complete the threatening activity).

\section{[TABLE 2][TABLE 3]}

No associations between heart rate variables and kinesiophobia or catastrophizing were observed (Table III). The percentage of the age-predicted maximal heart rate achieved at the completion of the stair climbing task ranged between 56 and 85, implying that the stair climbing to people with CFS corresponds to an exercise power at the frontier between endurance and resistive exercise.

\section{Are kinesiophobia and fatigue catastrophizing related to daily physical activity in CFS? \\ Kinesiophobia (i.e. both the TSK-CFS and TS-fatigue total score) and fatigue catastrophizing were unrelated to physical activity variables of the first day following stair climbing or the summed physical activity data of the 7 days following stair climbing ( $>0.01)$.}

\section{Validity of the TSK-CFS vs. TS-fatigue for measuring kinesiophobia in CFS The total scores of the TSK-CFS and TS-fatigue were closely and positively related to one another $(\rho=0.74 ; p<0.001)$, supporting the concurrent validity of both measures and suggesting that they measure the same underlying construct (i.e. kinesiophobia). Neither of the measures was related to symptom expectancies following stair climbing or the difference between anticipated and experienced symptoms following stair climbing ( $p>0.01$ ). As explained above, both measures were strongly related to stair climbing duration (Table III), supporting their criterion validity.}

\section{DISCUSSION}

\section{Threat control}

Based on the threat control analysis, it is concluded that stair climbing represents a threatening task to the people with CFS studied here. This is important as the study of fear (i.e. kinesiophobia or irrational fear of movement) requires a threatening stimulus. Hence, the outcome of the threat control analysis supports the internal validity of the study.

\section{Does symptom severity change following stair climbing in CFS?}

There was no change in symptom severity from baseline up to 7 days post-stair climbing. Hence, stair climbing did not trigger a symptom flare in the patients studied here. This is important as previous studies revealed that a submaximal exercise, or even a self-paced and physiologically limited bicycle or walking bout, triggers a severe relapse in patients with CFS [1-3,30,31]. The present study shows that people with CFS can easily perform a daily physical activity, perceived by themselves as threatening, without triggering symptom flares. This finding can be used to convince people with CFS of undertaking such a threatening task during treatment programs like graded activity or graded exposure [32]. It also underscores the importance of restructuring the beliefs of CFS patients about the relationship between activity and symptoms. 
Nijs, J., Meeuw, M., Heins, M., Knoop, H., Moorkens, G., Bleijenberg, G. Kinesiophobia, catastrophizing and anticipated symptoms before stair climbing in chronic fatigue syndrome: an experimental study. Disability and Rehabilitation: 2012, 34(15), 1299-1305

\section{Are symptom expectancies and kinesiophobia related to stair climbing performance in CFS?}

Previously, we reported that in Dutch people with CFS, performance during stair climbing is related to higher momentary and expected fatigue levels and focusing on bodily symptoms and fatigue catastrophizing influence expected fatigue levels [16]. The present study reveals that kinesiophobia and fatigue catastrophizing, but not symptom expectancies, are strongly related to stair climbing performance in Belgian people with CFS. These data underscore the importance of kinesiophobia and fatigue catastrophizing for performing threatening and physical demanding tasks in everyday life of people with CFS.

The major findings from our study of Dutch patients with CFS [16] are replicated in this study of Belgian patients with CFS. However, performance during stair climbing was unrelated to higher momentary and expected fatigue levels in Belgian people with CFS, which contradicts the findings from the Dutch sample. The observed differences between Dutch and Belgian patients with CFS might be due to a number of factors. First, cross-cultural differences might explain some of the discrepancies. Cognitions and beliefs might differ across countries, as they might vary depending on the local health care system or even the media coverage of the illness. A second possible explanation entails the applied study methods. Although care was taken to apply the same methodology in Belgium and the Netherlands, it was impossible to completely standardize the stair climbing protocol (i.e. the stairs differed in size, which might alter the severity of the task slightly). Third, CFS represents a heterogeneous disorder. This becomes particularly important in studies with a relatively small sample size. Fourth, although care was taken to standardize the instructions provided by the researchers in both centres, even small differences in verbal tone or expression might alter the patient's expectations. Finally, the Belgian patients with CFS, on an average, did not overestimate the symptom changes following stair climbing (as evidenced by the very small mean difference between anticipated and experienced fatigue/pain following stair climbing - Table II).

\section{Are kinesiophobia and fatigue catastrophizing related to daily physical activity in CFS?}

Previous studies suggested that high fatigue expectations are related to low physical activity levels in people with CFS [12], which suggests that people with CFS try to prevent symptoms by avoiding physical activity. The data from the present study indicate this to be the case for threatening physical activities such as stair climbing but not for physical activity in general. Neither kinesiophobia nor fatigue catastrophizing is associated with daily physical activity in patients with CFS. This is important for treatments aiming at increasing daily physical activity level in patients with CFS (as typically done in graded activity programs). From the data presented here, diminishing kinesiophobia and fatigue catastrophizing appears only relevant for targeting physical activities that are perceived as threatening, and not for increasing work-related or social (physical) activities. This suggests that clinicians should first identify physical activities that are perceived as threatening, and then restructure the patient's beliefs in relation to this threatening task specifically. Importantly, our results suggest that treatment for CFS should not address kinesiophobia or fatigue catastrophizing in general, or in relation to all sorts of physical activity. 
Nijs, J., Meeuw, M., Heins, M., Knoop, H., Moorkens, G., Bleijenberg, G. Kinesiophobia, catastrophizing and anticipated symptoms before stair climbing in chronic fatigue syndrom an experimental study. Disability and Rehabilitation: 2012, 34(15), 1299-1305

Besides psychological factors, immune factors are known to impact upon symptom increases following physical activity in people with CFS. People with CFS respond to an exercise challenge with an enhanced complement activation [5,30], increased oxidative stress [1] and a defective adaptive response to oxidative stress [33,34]. More specifically, the body of people with CFS produces insufficient antioxidants and heat-shock proteins to protect the cells against the deleterious effects of reactive oxygen species which are typically augmented following exercise [33,34].

\section{Validity of the TSK-CFS vs. TS-fatigue for measuring kinesiophobia in CFS}

This is the first study comparing two different measures for assessing kinesiophobia in people with CFS. While the TS-fatigue measures fatigue-related kinesiophobia, the TSK-CFS measures fear of worsening the overall symptom complex (including fatigue, pain, concentration difficulties, etc.) due to physical activity. Regretfully, the study findings do not allow to draw firm conclusions regarding the utility or validity of one measure over the other. Data in support of the validity (concurrent and criterion validity) of both measures were provided. Hence, this study adds to the body of evidence supporting the psychometric properties of the TSK-CFS. Indeed, extensive work supporting its internal consistency, test-retest reliability, congruent, convergent and content validity in CFS patients has been provided [10,18]. For the TS-fatigue, data supporting its internal consistency have been provided, and its construction was based on a varimax rotated factor analysis [16]. Thus, more work is required to identify the most convenient measure for assessing kinesiophobia in people with CFS.

At present, the use of both measures can be advocated. Clinicians can use the TSKCFS for assessing the fear of patients with CFS to exacerbate their symptoms (including pain, fatigue and brain fog) due to physical activities. Likewise, clinicians can use the TS-fatigue for assessing the fear of patients with CFS to exacerbate their fatigue level due to physical activities.

\section{Study strengths and limitations}

The use of a physical demanding task which everyone is familiar with, the established threat value of the task, the use of measures with established psychometric properties, the 1-week follow-up of symptom severity and real-time physical activity monitoring are important study strengths. The replication of some of the findings from the Dutch part of this international multicentre study [16] supports the validity of both studies for examining the role of kinesiophobia and catastrophizing in people with CFS. The sample size accounts for the a priori sample size calculation and generates a study power of 0.90 . On the other hand, the sample size was too low to enable the use of more sophisticated statistical methods like regression analysis and structural equation modelling. In addition, a clear experimental design would imply the cross-over randomization of patients to either a threatening or non-threatening physical activity.

\section{CONCLUSIONS}

In summary, kinesiophobia and fatigue catastrophizing are strongly associated to poorer stair climbing performance in Belgian people with CFS. These findings 
Nijs, J., Meeuw, M., Heins, M., Knoop, H., Moorkens, G., Bleijenberg, G. Kinesiophobia, catastrophizing and anticipated symptoms before stair climbing in chronic fatigue syndrom an experimental study. Disability and Rehabilitation: 2012, 34(15), 1299-1305

underscore the importance of kinesiophobia and fatigue catastrophizing for performing threatening and physical demanding tasks in everyday life of people with CFS. At the same time, these findings refute a cardinal role for kinesiophobia and fatigue catastrophizing in determining daily physical activity level of people with CFS.

Declaration of Interest: Mira Meeus is a postdoctoral research fellow of the Research Foundation Flanders (FWO). The other authors report no conflicts of interest.

\section{REFERENCES}

Jammes Y, Steinberg JG, Mambrini O, Brégeon F, Delliaux S. Chronic fatigue syndrome: assessment of increased oxidative stress and altered muscle excitability in response to incremental exercise. J Intern Med 2005;257:299-310.

Lapp CW. Exercise limits in chronic fatigue syndrome. Am J Med 1997;103:83-84.

Bazelmans E, Bleijenberg G, Voeten MJ, van der Meer JW, Folgering H. Impact of a maximal exercise test on symptoms and activity in chronic fatigue syndrome. J Psychosom Res 2005;59:201-208.

Black CD, O'connor PJ, McCully KK. Increased daily physical activity and fatigue symptoms in chronic fatigue syndrome. Dyn Med 2005;4:3.

Sorensen B, Streib JE, Strand M, Make B, Giclas PC, Fleshner M, Jones JF. Complement activation in a model of chronic fatigue syndrome. J Allergy Clin Immunol 2003;112:397403.

Ohashi K, Yamamoto Y, Natelson BH. Activity rhythm degrades after strenuous exercise in chronic fatigue syndrome. Physiol Behav 2002;77:39-44.

Kori SH, Miller RP, Todd DD. Kinesiophobia: a new view of chronic pain behavior. Pain Manag 1990; Jan/Feb:35-43.

Silver A, Haeney M, Vijayadurai P, Wilks D, Pattrick M, Main CJ. The role of fear of physical movement and activity in chronic fatigue syndrome. J Psychosom Res 2002;52:485-493.

Nijs J, Vanherberghen K, Duquet W, De Meirleir K. Chronic fatigue syndrome: lack of association between pain-related fear of movement and exercise capacity and disability. Phys Ther 2004;84:696-705.

Nijs J, De Meirleir K, Duquet W. Kinesiophobia in chronic fatigue syndrome: assessment and associations with disability. Arch Phys Med Rehabil 2004;85:1586-1592.

Gallagher AM, Coldrick AR, Hedge B, Weir WR, White PD. Is the chronic fatigue syndrome an exercise phobia? A case control study. J Psychosom Res 2005;58:367-373.

Vercoulen JH, Bazelmans E, Swanink CM, Fennis JF, Galama JM, Jongen PJ, Hommes O, et al. Physical activity in chronic fatigue syndrome: assessment and its role in fatigue. J Psychiatr Res 1997;31:661-673.

Sullivan MJL, Bishop SR, Pivik J. The Pain Catastrophizing Scale: development and validation. Psychol Assess 1995;7:524-532.

Petrie K, Moss-Morris R, Weinman J. The impact of catastrophic beliefs on functioning in chronic fatigue syndrome. J Psychosom Res 1995;39:31-37.

Nijs J, Van de Putte K, Louckx F, Truijen S, De Meirleir K. Exercise performance and chronic pain in chronic fatigue syndrome: the role of pain catastrophizing. Pain Med 2008;9:11641172.

Heins M, Knoop H, Nijs J, Meeus M, Moorkens G, Bleijenberg G. The influence of symptom expectancies on stair climbing performance in chronic fatigue syndrome: evidence for the role of symptom focusing and catastrophizing. Submitted.

Cohen J. Statistical power analysis for the behavioural sciences. 2nd ed. Hillsdale, $\mathrm{NJ}$ : Lawrence Erlbaum Associates; 1988

Nijs J, Thielemans A. Kinesiophobia and symptomatology in chronic fatigue syndrome: a psychometric study of two questionnaires. Psychol Psychother 2008;81:273-283. 
Nijs, J., Meeuw, M., Heins, M., Knoop, H., Moorkens, G., Bleijenberg, G. Kinesiophobia, catastrophizing and anticipated symptoms before stair climbing in chronic fatigue syndrom an experimental study. Disability and Rehabilitation: 2012, 34(15), 1299-1305

Nijs J, Aerts A, De Meirleir K. Generalized joint hypermobility is more common in chronic fatigue syndrome than in healthy control subjects. J Manipulative Physiol Ther 2006;29:3239.

Ware JE, Snow KK, Kosinski M, Gandek B. SF-36 Health Survey manual and interpretation guide. Boston: The Health Institute; 1993.

Wells KB, Stewart A, Hays RD, Burnam MA, Rogers W, Daniels M, Berry S, et al. The functioning and well-being of depressed patients. Results from the Medical Outcomes Study. JAMA 1989;262:914-919.

McHorney CA, Ware JE Jr, Lu JF, Sherbourne CD. The MOS 36-item Short-Form Health Survey (SF-36): III. Tests of data quality, scaling assumptions, and reliability across diverse patient groups. Med Care 1994;32:40-66.

Nijs J, Vaes P, Van Hoof E, De Becker P, McGregor N, De Meirleir K. Activity limitations and participation restrictions in patients with chronic fatigue syndrome - construction of a disease specific questionnaire. J Chronic Fatigue Syndr 2002;10:3-23.

Vercoulen JH, Swanink CM, Fennis JF, Galama JM, van der Meer JW, Bleijenberg G. Dimensional assessment of chronic fatigue syndrome. J Psychosom Res 1994;38:383392.

Vercoulen JH, Hommes OR, Swanink CM, Jongen PJ, Fennis JF, Galama JM, van der Meer JW, Bleijenberg $\mathrm{G}$. The measurement of fatigue in patients with multiple sclerosis. A multidimensional comparison with patients with chronic fatigue syndrome and healthy subjects. Arch Neurol 1996;53:642-649.

Vercoulen JH, Alberts M, Bleijenberg G. De checklist individual strength (CIS). Gedragstherapie 1999;32:131-136.

Vlaeyen JW, Kole-Snijders AM, Boeren RG, van Eek H. Fear of movement/(re)injury in chronic low back pain and its relation to behavioral performance. Pain 1995;62:363-372.

Jacobsen PB, Azzarello LM, Hann DM. Relation of catastrophizing to fatigue severity in women with breast cancer. Cancer Res Ther Control 1999;8:155-64.

Finn KJ, Specker B. Comparison of Actiwatch activity monitor and Children's Activity Rating Scale in children. Med Sci Sports Exerc 2000;32:1794-1797.

Nijs J, Van Oosterwijck J, Meeus M, Lambrecht L, Metzger K, Frémont M, Paul $\mathrm{L}$. Unravelling the nature of postexertional malaise in myalgic encephalomyelitis/chronic fatigue syndrome: the role of elastase, complement C4a and interleukin-1beta. J Intern Med 2010;267:418-435.

Nijs J, Almond F, De Becker P, Truijen S, Paul L. Can exercise limits prevent post-exertional malaise in chronic fatigue syndrome? An uncontrolled clinical trial. Clin Rehabil 2008;22:426-435.

Vlaeyen JW, de Jong J, Geilen M, Heuts PH, van Breukelen G. The treatment of fear of movement/(re)injury in chronic low back pain: further evidence on the effectiveness of exposure in vivo. Clin J Pain 2002;18:251-261.

Jammes Y, Steinberg JG, Delliaux S, Brégeon F. Chronic fatigue syndrome combines increased exercise-induced oxidative stress and reduced cytokine and Hsp responses. J Intern Med 2009;266:196-206.

Thambirajah AA, Sleigh K, Stiver HG, Chow AW. Differential heat shock protein responses to strenuous standardized exercise in chronic fatigue syndrome patients and matched healthy controls. Clin Invest Med 2008;31:E319-E327.

Fukuda K, Straus SE, Hickie I, Sharpe MC, Dobbins JG, Komaroff A. The chronic fatigue syndrome: a comprehensive approach to its definition and study. International Chronic Fatigue Syndrome Study Group. Ann Intern Med 1994;121:953-959.

\section{FIGURES AND TABLES}

Figure 1. Flow diagram of the study. CDC, center for disease control and prevention; CFS, chronic fatigue syndrome; SF-36, Medical Outcomes Short Form 36 Health Status Survey; shortened TS-fatigue, shortened version of the Tampa scale for fear of fatigue; CIS, Checklist 
Nijs, J., Meeuw, M., Heins, M., Knoop, H., Moorkens, G., Bleijenberg, G. Kinesiophobia, catastrophizing and anticipated symptoms before stair climbing in chronic fatigue syndrome:

Individual Strength; TSK-CFS, Tampa scale kinesiophobia version CFS;

J-FCS, Jacobson-Fatigue Catastrophizing Scale.

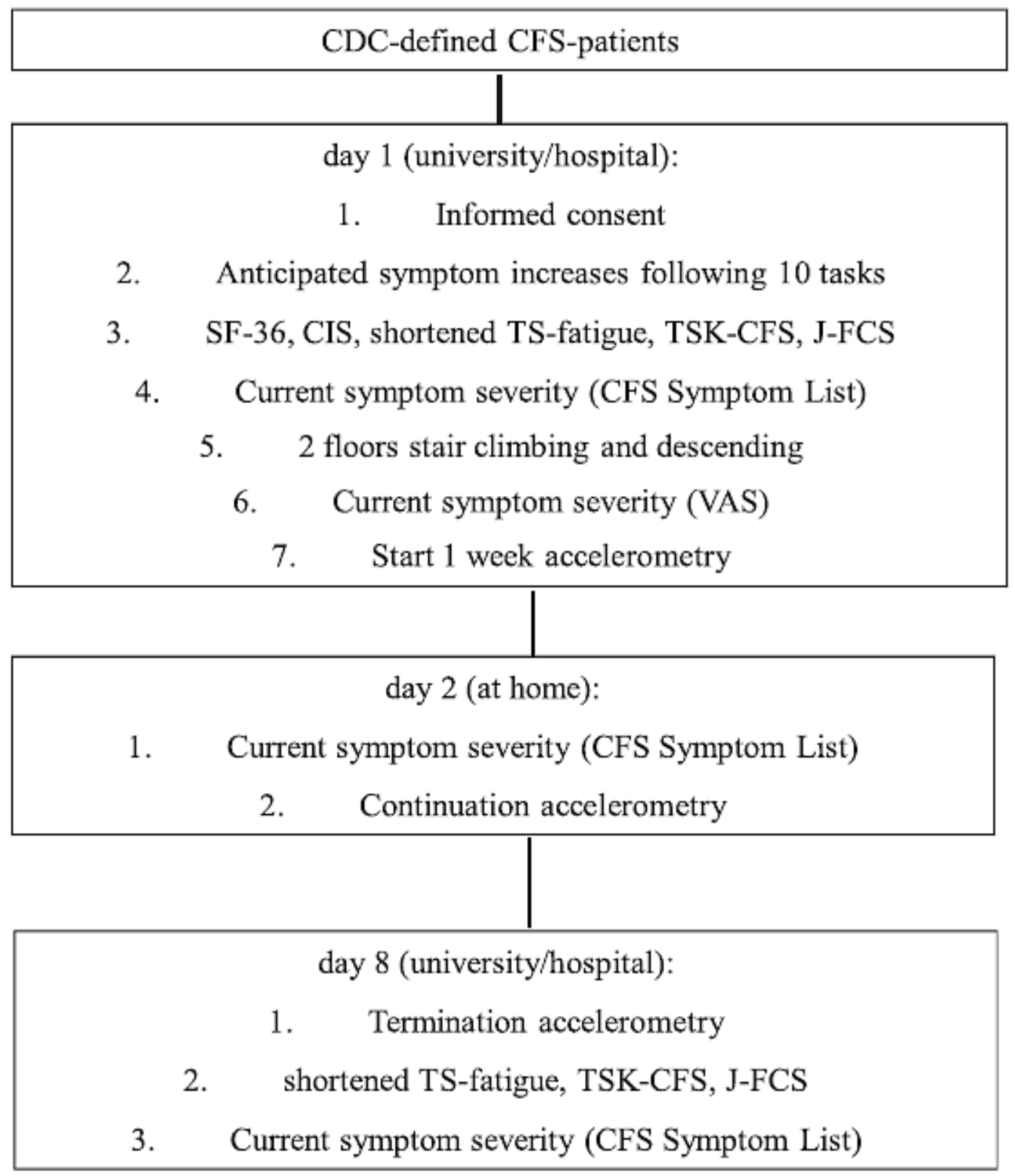


Nijs, J., Meeuw, M., Heins, M., Knoop, H., Moorkens, G., Bleijenberg, G. Kinesiophobia, catastrophizing and anticipated symptoms before stair climbing in chronic fatigue syndrome: an experimental study. Disability and Rehabilitation: 2012, 34(15), 1299-1305

Table I. Descriptive statistics of the study participants (21 women with chronic fatigue syndrome) at baseline (day 1 of the experiment).

\begin{tabular}{lc}
\hline Descriptive variable & $\begin{array}{c}\text { Mean } \pm \text { standard } \\
\text { deviation }\end{array}$ \\
\hline Age (years) & $44.7 \pm 10.5$ \\
Body weight (kg) & $69.6 \pm 13.8$ \\
Body length (cm) & $165.2 \pm 7.7$ \\
Illness duration (months) & $89.9 \pm 52.8$ \\
CIS fatigue & $43.9 \pm 10.9$ \\
CIS concentration & $27.1 \pm 6.0$ \\
CIS motivation & $15.4 \pm 6.3$ \\
CIS activity & $13.7 \pm 4.3$ \\
SF-36 physical functioning & $51.4 \pm 19.8$ \\
SF-36 role limitations due to physical functioning & $30.9 \pm 33.5$ \\
SF-36 bodily pain & $48.1 \pm 21.4$ \\
SF-36 general health perception & $47.4 \pm 18.6$ \\
SF-36 vitality & $39.5 \pm 17.5$ \\
SF-36 social functioning & $48.3 \pm 22.4$ \\
SF-36 role limitations due to emotional problems & $71.4 \pm 38.4$ \\
SF-36 mental health & $68.6 \pm 16.2$ \\
\hline CIS, Checklist Individual Strength; SF-36, Medical Outcomes Short Form 36 Health \\
Status Survey. & \\
\hline
\end{tabular}

Table II. Descriptive statistics of the kinesiophobia and stair climbing data $(\mathrm{n}=21)$.

\begin{tabular}{lr}
\hline Descriptive variable & Mean \pm standard deviation \\
\hline Stair climbing duration (minutes) & $85.2 \pm 20.0$ \\
Heart rate at completion of stair climbing (beats per minute) & $120.2 \pm 17.6$ \\
Heart rate increase during stair climbing (beats per minute) & $35.6 \pm 12.5$ \\
Percentage of age-predicted maximal heart rate achieved at the completion of stair climbing & $68.6 \pm 9.2$ \\
Experienced fatigue at baseline (mm) & $54.7 \pm 21.6$ \\
Anticipated fatigue following stair climbing (mm) & $59.9 \pm 29.0$ \\
Experienced fatigue following stair climbing (mm) & $59.1 \pm 27.0$ \\
Difference between anticipated and experienced fatigue following stair climbing (mm) & $-2.1 \pm 32.5$ \\
Experienced pain at baseline (mm) & $49.1 \pm 29.7$ \\
Anticipated pain following stair climbing (mm) & $49.3 \pm 27.4$ \\
Experienced pain following stair climbing (mm) & $46.9 \pm 28.3$ \\
Difference between anticipated and experienced pain following stair climbing (mm) & $0.5 \pm 27.9$ \\
TSK-CFS & $32.0 \pm 5.3$ \\
TS-fatigue & $13.0 \pm 4.6$ \\
Fatigue catastrophizing & $0.9 \pm 0.5$
\end{tabular}

TSK-CFS, Tampa scale kinesiophobia version chronic fatigue syndrome; TS-fatigue, Tampa scale fatigue. 
Nijs, J., Meeuw, M., Heins, M., Knoop, H., Moorkens, G., Bleijenberg, G. Kinesiophobia, catastrophizing and anticipated symptoms before stair climbing in chronic fatigue syndrome: an experimental study. Disability and Rehabilitation: 2012, 34(15), 1299-1305

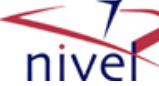

Table II. Descriptive statistics of the kinesiophobia and stair climbing data $(\mathrm{n}=21)$.

Descriptive variable Mean \pm standard deviation

Stair climbing duration (minutes) $85.2 \pm 20.0$

Heart rate at completion of stair climbing (beats per minute)

$120.2 \pm 17.6$

Heart rate increase during stair climbing (beats per minute)

$35.6 \pm 12.5$

Percentage of age-predicted maximal heart rate achieved at the completion of stair climbing

$68.6 \pm 9.2$

Experienced fatigue at baseline $(\mathrm{mm})$

$54.7 \pm 21.6$

Anticipated fatigue following stair climbing $(\mathrm{mm})$

$59.9 \pm 29.0$

Experienced fatigue following stair climbing $(\mathrm{mm})$

$59.1 \pm 27.0$

Difference between anticipated and experienced fatigue following stair climbing ( $\mathrm{mm}$ )

$-2.1 \pm 32.5$

Experienced pain at baseline $(\mathrm{mm})$

$49.1 \pm 29.7$

Anticipated pain following stair climbing ( $\mathrm{mm}$ )

$49.3 \pm 27.4$

Experienced pain following stair climbing $(\mathrm{mm})$

$46.9 \pm 28.3$

Difference between anticipated and experienced pain following stair climbing $(\mathrm{mm})$

$0.5 \pm 27.9$

TSK-CFS

$32.0 \pm 5.3$

TS-fatigue

$13.0 \pm 4.6$

Fatigue catastrophizing

$0.9 \pm 0.5$

TSK-CFS, Tampa scale kinesiophobia version chronic fatigue syndrome; TS-fatigue, Tampa scale fatigue. 